\title{
Snapshots of a hybrid transcription factor in the Hippo pathway
}

\author{
Xuelian Luo ${ }^{\bowtie}$ \\ Department of Pharmacology, University of Texas Southwestern Medical Center, 6001 Forest Park Road, Dallas, TX 75390, USA \\ $\triangle$ Correspondence: xuelian.luo@utsouthwestern.edu \\ Received July 30, 2010 Accepted August 16, 2010
}

\begin{abstract}
The Hippo pathway plays key roles in animal development. It suppresses tumorigenesis by controlling the transcription of the target genes that are critical for cell proliferation and apoptosis. The transcriptional coactivator YAP is the major downstream effector of the Hippo signaling. Upon extracellular stimulation, a kinase cascade in the Hippo pathway phosphorylates YAP and promotes its cytoplasmic sequestration by 14-3-3 and ubiquitin-dependent degradation. When the Hippo pathway is turned off, YAP (which lacks a DNA-binding domain) is dephosphorylated and translocates to the nucleus, where it associates with the transcription factor TEAD to form a functional heterodimeric transcription factor and to promote the expression of the Hipporesponsive genes. Recently, structures of the YAPbinding domain of TEAD alone or in complex with YAP have revealed the atomic details of the TEAD-YAP interaction. Here, I review these exciting advances, propose a strategy for targeting the TEAD-YAP interaction using small molecules, and suggest potential mechanisms by which phosphorylation and 14-3-3 binding regulate the cytoplasmic retention of YAP.
\end{abstract}

\section{KEYWORDS Hippo, TEAD, YAP, structure, phosphor-}

ylation, cancer

\section{OVERVIEW OF THE HIPPO PATHWAY}

Tissue homeostasis is vital for the normal development and physiology of multicellular organisms. Best characterized in Drosophila, the Hippo signaling pathway is an evolutionarily conserved pathway for organ size control and tumor suppression in metazoans (Edgar, 2006; Harvey and Tapon, 2007; Pan, 2007; Badouel et al., 2009; Oh and Irvine, 2010;
Zhao et al., 2010a). The core components of this tumor suppressor pathway were identified through mosaic genetic screens for overgrowth mutants in Drosophila. These include the Ste20 family kinase Hippo (Hpo), the WW domaincontaining adaptor protein Salvador (Sav), the NDR family protein kinase Warts (Wts), and the NDR family kinase activator Mats (Justice et al., 1995; Xu et al., 1995; KangoSingh et al., 2002; Tapon et al., 2002; Harvey et al., 2003; Wu et al., 2003; Lai et al., 2005) (Fig. 1).

These four tumor suppressors form two heterodimeric kinase complexes: Hpo-Sav and Wts-Mats. The activation of $\mathrm{Hpo}$ requires autophosphorylation of its activation loop in the kinase domain. Hpo binds Sav directly and promotes Sav phosphorylation. The Hpo-Sav complex phosphorylates both Wts and Mats, therefore promotes autophosphorylation of Wts in its activation loop and further activates the Wts-Mats complex by strengthening their association. The activated Wts-Mats complex in turn phosphorylates the transcriptional coactivator Yorki (Yki), which then binds to 14-3-3 proteins, resulting in its cytoplasmic sequestration and inactivation (Huang et al., 2005; Dong et al., 2007; Zhao et al., 2007; Oh and Irvine, 2008; Ren et al., 2010). When the Hippo pathway is inactivated, Yki is dephosphorylated and translocates to the nucleus. Because of lacking a DNA binding domain, Yki has to interact with other transcription factors for gene expression regulation. Scalloped $(S d)$ is the first transcription factor (which contains a sequence-specific DNA binding domain) identified to form a functional, heterodimeric transcription factor with Yki (Wu et al., 2008). The Yki-Sd hybrid transcription factor mediates the transcription of Hipporesponsive genes, such as the cell cycle gene cyclin $E$, the anti-apoptotic gene Diap1, and the microRNA Bantam, thereby promoting cell growth and proliferation and inhibiting apoptosis (Nolo et al., 2006; Thompson and Cohen, 2006).

The upstream regulators of the Hippo pathway are less understood. Over the past few years, additional tumor 

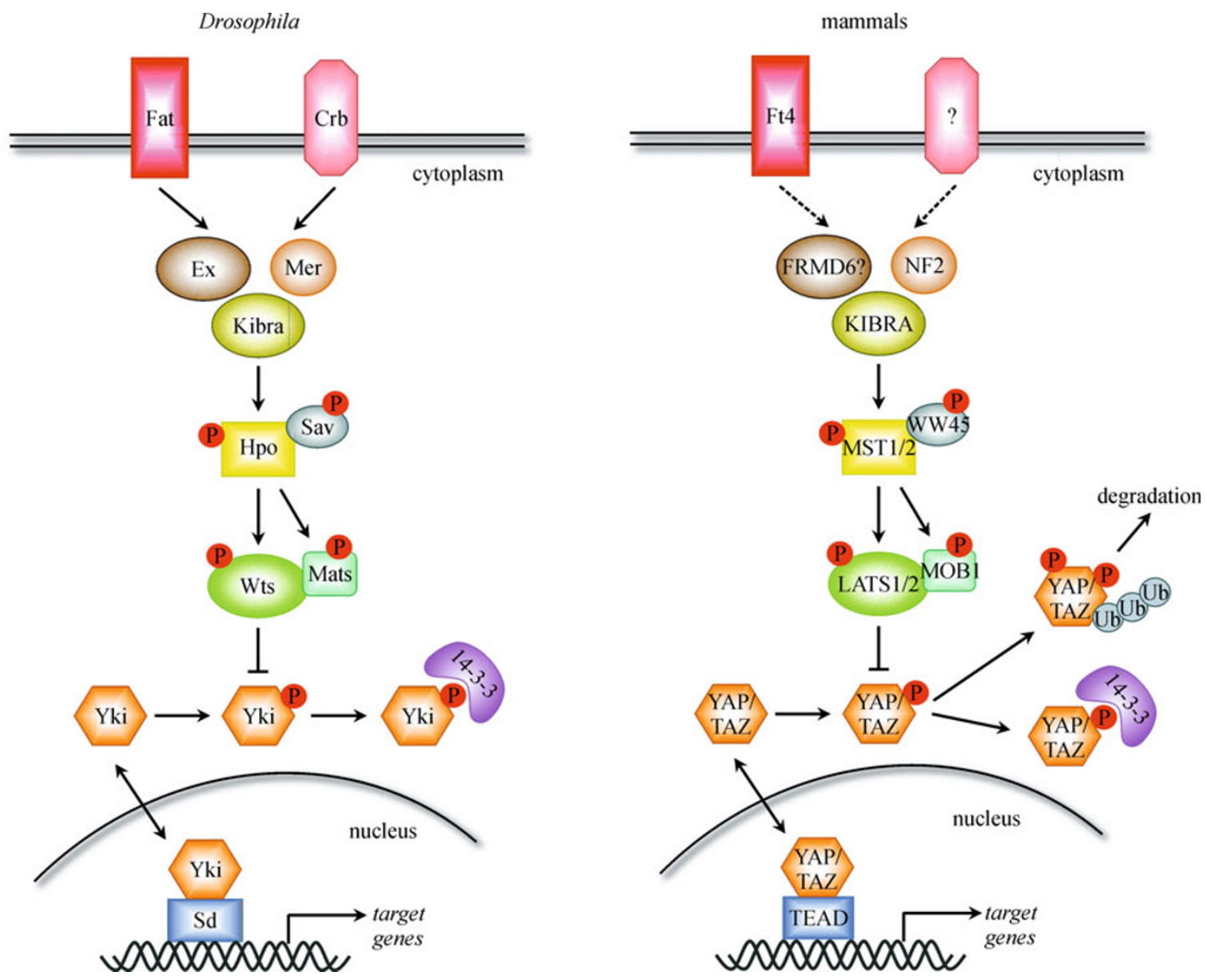

Figure 1. The Hippo signaling pathway in Drosophila and mammals. Interactions among different components of the Hippo pathway are shown. Pointed arrows indicate positive regulations and blocked arrows indicate negative regulations. In Drosophila, upon activation, the Hippo pathway transduces extracellular signals through cell surface receptors (Fat and Crb) to apical membrane-associated cytoplasmic proteins (Ex, Mer and Kibra) to activate the core kinase cascade (Hpo, Sav, Wts and Mats). The kinase cascade phosphorylates the transcription coactivator Yki and causes its cytoplasmic sequestration and inactivation by 14-3-3. Unphosphorylated Yki translocates to the nucleus and binds to transcription factor Sd to turn on the expression of target genes. The Hippo pathway is conserved in mammals. The corresponding Drosophila homologs of the Hippo pathway in mammals are drawn and colored the same. The upstream of mammalian Hippo pathway is still not clear. Furthermore, phosphorylation of YAP/TAZ by the mammalian Hippo pathway provides a novel mechanism for regulating YAP/TAZ activity through protein degradation.

suppressor genes in Drosophila have been linked to Hippo signaling and have unveiled an intricate upstream regulatory scheme of this pathway (Grusche et al., 2010). These include two FERM domain-containing cytoskeletal proteins, Expanded (Ex) and Merlin (Mer), and the WW- and C2-domain-containing protein Kibra (Baumgartner et al., 2010; Genevet et al., 2010; Yu et al., 2010) (Fig. 1). These apical membrane-associated, cytoplasmic proteins have been suggested to form a functional complex and regulate some components of the Hippo pathway. The Ex-Mer-Kibra complex or subcomplexes may activate Hpo-Sav through direct interactions. The atypical cadherin Fat $(\mathrm{Ft})$ and the apical transmembrane protein Crumbs (Crb) function as tumor suppressors and have been implicated as potential cell surface receptors for Hippo signaling (Grzeschik et al., 2010; Ling et al., 2010; Robinson et al., 2010). Crb and Ex directly bind to each other, linking a transmembrane protein to an apical component of the Hippo pathway.

The Hippo pathway is highly conserved in mammals (Edgar, 2006; Harvey and Tapon, 2007; Pan, 2007; Reddy and Irvine, 2008; Zeng and Hong, 2008; Zhao et al., 2008a, 2010a; Zhang et al., 2009b) (Fig. 1). All of its core components in Drosophila have mammalian homologs, including MST1/2 for Hpo, WW45 for Sav, LATS1/2 for Wts, MOB1 for Mats, and YAP/TAZ for Yki. Expression of human MST2, LATS1, MOB1 and YAP proteins in fly functionally rescues the phenotypes of 
the corresponding Drosophila mutants, indicating that these genes are functionally conserved through evolution (Tao et al., 1999; Wu et al., 2003; Huang et al., 2005; Lai et al., 2005; Dong et al., 2007; Zhao et al., 2007; Wu et al., 2008). TEAD is the mammalian homolog of Sd and form functional, hybrid transcription factors with YAP/TAZ (Cao et al., 2008; Ota and Sasaki, 2008; Wu et al., 2008; Zhao et al., 2008b). The human or mouse genome each encodes four closely related TEAD proteins, TEAD1-4. When the mammalian Hippo pathway is activated, the MST-WW45 complex phosphorylates and activates the LATS-MOB1 complex. The activated LATSMOB1 complex phosphorylates YAP and promotes its association with 14-3-3 proteins and cytoplasmic retention, thus preventing the formation of the YAP-TEAD hybrid transcription factors. Moreover, phosphorylation of YAP by LATS promotes its subsequent phosphorylation by $\mathrm{CK} 1 \delta / \varepsilon$ (Zhao et al., 2010b). The hyperphosphorylated YAP is recognized and ubiquitinated by the $\mathrm{SCF}^{\beta T R C P}$ E3 ligase, leading to YAP degradation. As in Drosophila, when the Hippo pathway is turned off in mammals, YAP translocates to the nucleus, where it forms functional, heterodimeric transcription factors with TEAD and mediates the expression of proproliferative genes.

Homologs of the upstream components in the Drosophila Hippo pathway exist in mammals, including FRMD6 for Ex, NF2 for Mer, KIBRA for Kibra, and Fat4 (Ft4) for Fat (Grusche et al., 2010). It remains to be demonstrated, however, whether these proteins indeed regulate Hippo signaling in mammals. Moreover, the target genes of the mammalian Hippo pathway are not identical as those found in the fly. So far, only the connective tissue growth factor (CTGF) has been shown to be a direct target gene induced by YAP-TEAD to promote tissue overgrowth (Zhao et al., 2007). YAP and TAZ also interact with other transcription factors to mediate Hippo signaling (Hao et al., 2008; Varelas et al., 2008; Zhao et al., 2008b; Alarcón et al., 2009; Zhang et al., 2009a; Oh and Irvine, 2010). Thus, the Hippo pathway in mammals is much more complex, with built-in redundancy to ensure tissue homeostasis. In addition, distinct mechanisms may exist to control the organ-specific tissue growth.

The Hippo signaling pathway suppresses tumor formation. Dysregulation of the Hippo pathway has been implicated in human tumorigenesis. Mutations of the pathway components, such as NF2, WW45 and MOB1, have been linked to several types of human cancers (Tapon et al., 2002; Lai et al., 2005; Asthagiri et al., 2009). Downregulation of MST1/2, LATS1/2 and MOB1 has been observed in human sarcomas and various cancers (Hisaoka et al., 2002; Jiménez-Velasco et al., 2005; Takahashi et al., 2005; Kosaka et al., 2007; Minoo et al., 2007; Seidel et al., 2007). The main output of the Hippo pathway is to suppress the function of YAP by regulating its nuclear translocation and stability. YAP has been reported as a candidate oncogene in the human chromosome 11q22 amplicon, which is amplified in human hepatocellular carcinomas (HCC) and breast cancers (Overholtzer et al., 2006; Zender et al., 2006). The YAP protein is frequently overexpressed in several human cancers (Overholtzer et al., 2006; Zender et al., 2006; Zhao et al., 2007; Steinhardt et al., 2008; Liu et al., 2010).

The TEAD proteins are major partners of YAP and are required for the YAP-mediated gene expression program that promotes cell proliferation and inhibits apoptosis (Zhao et al., 2008b). Thus, understanding how TEAD interacts with YAP will provide insights into how the Hippo pathway regulates the YAP-TEAD transcription factors and may lead to strategies that control the oncogenic activity of YAP in tumor cells. In this review, I summarize the biochemical and structural data on the function and regulation of the TEAD-YAP hybrid transcription factor, and propose a model to explain how YAP phosphorylation might differentially regulate its binding to 143-3 or TEAD.

\section{STRUCTURES OF YAP WW DOMAINS AND TEAD DNA BINDING DOMAIN}

Human YAP protein contains an $\mathrm{N}$-terminal TEAD binding domain (TBD), two WW domains, and a C-terminal acidic transactivation domain (Fig. 2A). The WW domain consists of about 30 amino acids with two highly conserved tryptophan residues. Similar to $\mathrm{SH} 3$ domains, WW domains are proteininteracting modules frequently found in diverse, intracellular signaling proteins, and bind to proline-rich motifs with dissociation constants in the $\mu \mathrm{M}$ range. The structure of one of the WW domains of YAP in complex with a PPXY motifcontaining peptide was determined first (Macias et al., 1996). It contains a $\beta$-sheet with three anti-parallel strands. The PPXY peptide binds on the concave side of the $\beta$-sheet. The structure nicely explains the specificity of the YAP WW domain toward PPXY motifs.

The role of WW domains in YAP appears to be complicated, however. They are required for both cytoplasmic retention and transcriptional activity of YAP (Oh and Irvine, 2010; Zhao et al., 2010a). On the one hand, the WW domains of YAP bind to PPXY motifs in LATS, enabling efficient LATSmediated phosphorylation of YAP and its cytoplasmic retention (Hao et al., 2008; Oka et al., 2008). On the other hand, the WW domains of YAP interact with PPXY-containing transcription factors in the nucleus to promote gene expression in a cell-type-dependent manner (Zhao et al., 2009). Finally, YAP has an $\mathrm{N}$-terminal proline-rich region (Fig. $2 \mathrm{~A}$ ). It will be interesting to test whether that this $\mathrm{N}$-terminal region of YAP binds to its own WW domains intramolecularly to autoinhibit the function of WW domains.

All four human TEAD proteins have an $\mathrm{N}$-terminal TEA DNA binding domain and a C-terminal YAP binding domain (YBD). The structure of the TEA DNA binding domain of TEAD proteins was determined (Anbanandam et al., 2006). It consists of a three-helix bundle with a homeodomain fold. 
A

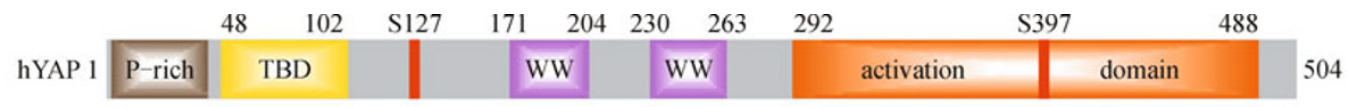

40 114 217

hTEAD2 1

TEA

YBD
447

B

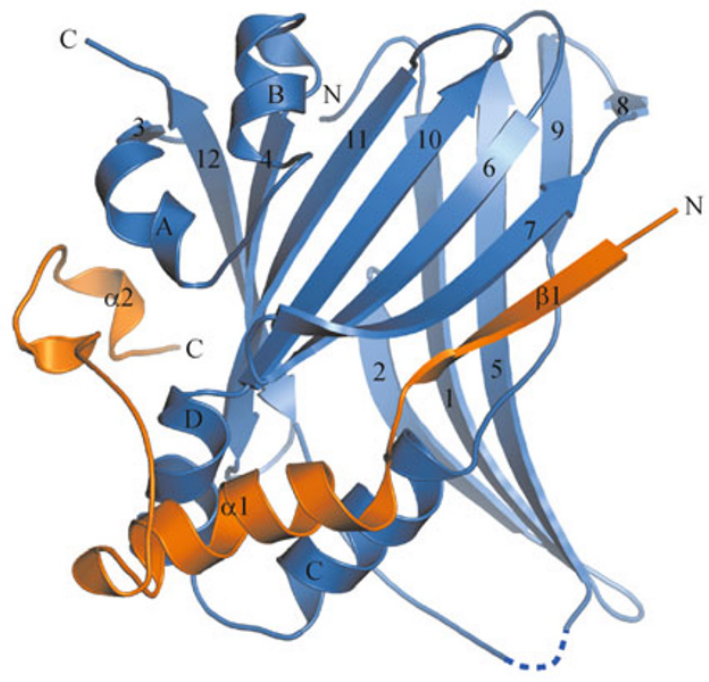

D

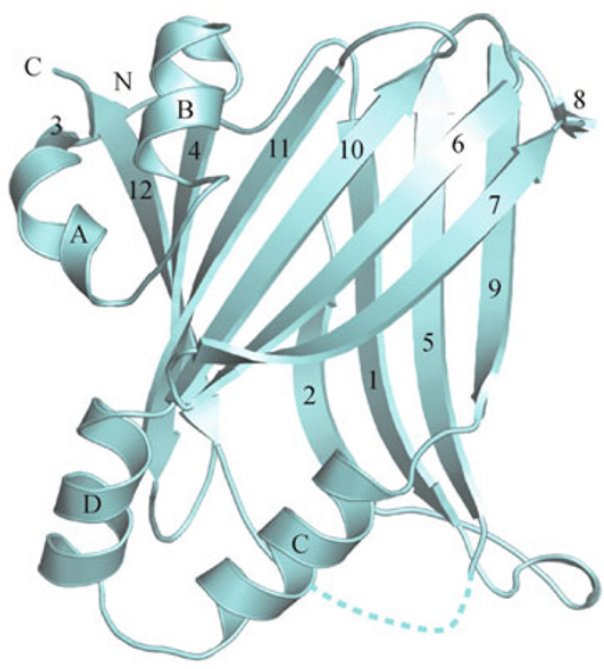

C

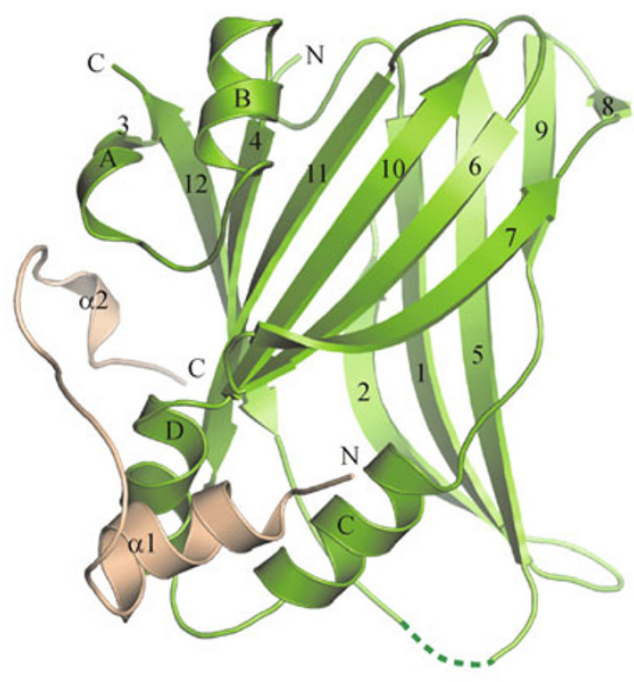

E



Figure 2. Structures of TEAD/YAP interaction. (A) Schematic drawing of the domain organization for human YAP and TEAD2 proteins. The residue numbers for different domain boundaries are labeled. P-rich: proline-rich region; TBD: TEAD binding domain; TEA: DNA binding TEA domain; YBD: YAP binding domain. (B) Ribbon diagram of hTEAD1-YAP complex (PDB ID code 3KYS). TEAD1 YBD is colored blue, and YAP TBD is colored orange. The N/C-termini and secondary structure elements are labeled. (C) Ribbon diagram of mTEAD4-YAP complex (PDB ID code 3JUA). TEAD4 YBD is colored green, and YAP TBD is colored wheat. The $\mathrm{N} / \mathrm{C}$-termini and secondary structure elements are labeled. (D) Ribbon diagram of hTEAD2 YBD (PDB ID code 3L15). The molecule is colored cyan. The N/C-termini and secondary structure elements are labeled. (E) Superposition of hTEAD1-YAP and hTEAD2. All structural figures were generated with PyMOL (http://www.pymol.org). 
Based on biochemical and NMR studies, it has been suggested that the TEA domain specifically recognizes the promoter regions of its target genes through interactions between its $\mathrm{C}$-terminal $\mathrm{H} 3$ helix and the major groove of DNA.

\section{STRUCTURES OF THE YAP-TEAD INTERACTION}

The YAP-TEAD heterodimeric transcription factor represents the first well-characterized hybrid transcription system. TEAD provides sequence-specific DNA binding to the promoters of the target genes while the YAP transactivation domain helps to recruit other components of the transcriptional machinery to initiate gene transcription. This elegant system requires the cooperativity between YAP and TEAD for function and increases the complexity of regulation by other cellular partners. Recent structural studies have revealed the interactions between YAP and TEAD in atomic details (Chen et al., 2010; Li et al., 2010; Tian et al., 2010).

The crystal structure of the YAP binding domain (YBD) of human TEAD2 showed that the molecule adopted an immunoglobulin (IgG)-like fold with two $\beta$-sheets packing against each other to form a $\beta$-sandwich (Fig. 2). One $\beta$-sheet contains five anti-parallel strands, including $\beta 1, \beta 2, \beta 5, \beta 8$ and $\beta 9$, while the other contains seven parallel and anti-parallel strands, including $\beta 3, \beta 4, \beta 6, \beta 7$, and $\beta 10-12$. In addition to the two main $\beta$ sheets, TEAD2 YBD contains two helix-turnhelix motifs that are absent in the IgG fold. One helix-turnhelix motif consists of $\alpha \mathrm{A}$ and $\alpha \mathrm{B}$, and connects $\beta 3$ and $\beta 4$. This motif, along with the $\beta 2-\beta 3$ loop, encircles the $C$-terminal $\beta 12$ strand, forming an unusual pseudo-knot structure. The second helix-turn-helix motif consists of $\alpha C$ and $\alpha D$, and connects $\beta 9$ and $\beta 10$.

The crystal structures of two TEAD-YAP complexes have also been solved, including human TEAD1 YBD bound to human YAP TBD, and mouse TEAD4 YBD bound to mouse YAP TBD. In the two TEAD-YAP complexes, the structures of TEAD YBD are highly similar to that of TEAD2 YBD in the absence of YAP (Fig. 2), indicating that YAP binds to a preformed binding pocket on TEAD and does not induce substantial conformational changes of TEAD. In both cases, YAP TBD occupies very similar positions, wrapping around an extended surface on TEAD and forming multiple contacts between the main chain amides and side chains of residues in helices $\alpha A, \alpha C$ and $\alpha D$, and strands $\beta 3, \beta 4, \beta 7, \beta 11$ and $\beta 12$ (Fig. 2).

In the hTEAD1-YAP structure, there are three interfaces between TEAD and YAP. In interface 1 , the $N$-terminal $\beta$ strand of YAP forms an edge-on interaction with $\beta 7$ of TEAD. Interface 2 is formed by $\alpha 1$ of YAP nestling in the hydrophobic groove between helices $\alpha C$ and $\alpha D$ of TEAD. Interface 3 lies between the $\alpha 2$ region of YAP and a pocket formed by helices $\alpha A$ and $\alpha D$, and strands $\beta 3, \beta 4, \beta 11$ and $\beta 12$ of TEAD. The mTEAD4-YAP structure reveals that the proteins interact through the two latter interfaces. The edge-on interaction between TEAD1 $\beta 7$ and the $N$-terminal $\beta 1$ of the YAP TBD is absent.

Based on mutagenesis data, interfaces 1 and 2 are not important for YAP binding because mutations of residues in these interfaces have little effect on the TEAD-YAP interaction. For example, a YAP fragment that lacks strand $\beta 1$ (interface 1) and helix $\alpha 1$ (interface 2) still binds to TEAD. By contrast, mutations of the residues at interface 3 dramatically weaken or abolish the TEAD-YAP interaction, indicating that this interface contributes most of the binding energy between TEAD and YAP (Fig. 3). A rare eye disease-causing mutation, $\mathrm{Y} 421 \mathrm{H}$ in TEAD1, that is known to disrupt the TEAD-YAP interaction is located at interface 3 (Kitagawa, 2007). TEAD Y421 forms a hydrogen bond with YAP S94, mutation of which also disrupts the TEAD-YAP interaction. Furthermore, among all structure-based mutants of TEAD, only 12 mutants lose binding to YAP, and they are located at interface 3 (Fig. $3 \mathrm{~A})$. Correspondingly, mutations of residues in the a2 region of YAP, which lie at interface 3, diminish TEAD binding. Collectively, interface 3 provides a major anchoring point for YAP binding to TEAD and likely contributes most of the binding energy between the two proteins.

Given the relatively small energetic contributions from interfaces 1 and 2, why then do TEAD proteins use such extensive interfaces to interact with YAP? I envision two nonexclusive possibilities. In the first possibility, these weak binding interfaces might contribute to the binding specificities between YAP/TAZ and TEAD1-4 proteins. For example, a PXXФP motif in the loop connecting $\alpha 1$ and $\alpha 2$ of YAP is important for TEAD4 binding and for the transforming activity of YAP. However, this PXXФP motif is not conserved in TAZ and is obviously not involved in mediating TAZ binding to TEAD proteins. Therefore, the extensive interface between YAP and TEAD affords an opportunity to fine-tune the binding specificity among the various YAP/TAZ and TEAD homologs. In the second possibility, interfaces 1 and 2 might allow TEAD proteins to disfavor certain residues at key positions in potential ligands, thus contributing to ligand specificity through negative selection. For example, proteins with short motifs that resemble the YAP sequence that binds at interface 3 may contain residues that generate destabilizing interactions at interfaces 1 and 2 . These proteins will not bind to TEAD. This type of negative selection has been observed in other protein-protein interactions, such as the interaction among histone binding modules and histone tails (Couture et al., 2007).

Regardless of the reason, the fact that interface 3 contributes most of the binding energy between YAP and TEAD suggests a feasible strategy for disrupting the YAPTEAD interaction with small molecules. TEAD has a conspicuous, preexisting surface pocket at this interface. Chemical compounds that bind to this pocket may disrupt the YAP-TEAD interactions. These compounds may kill cancer 
A

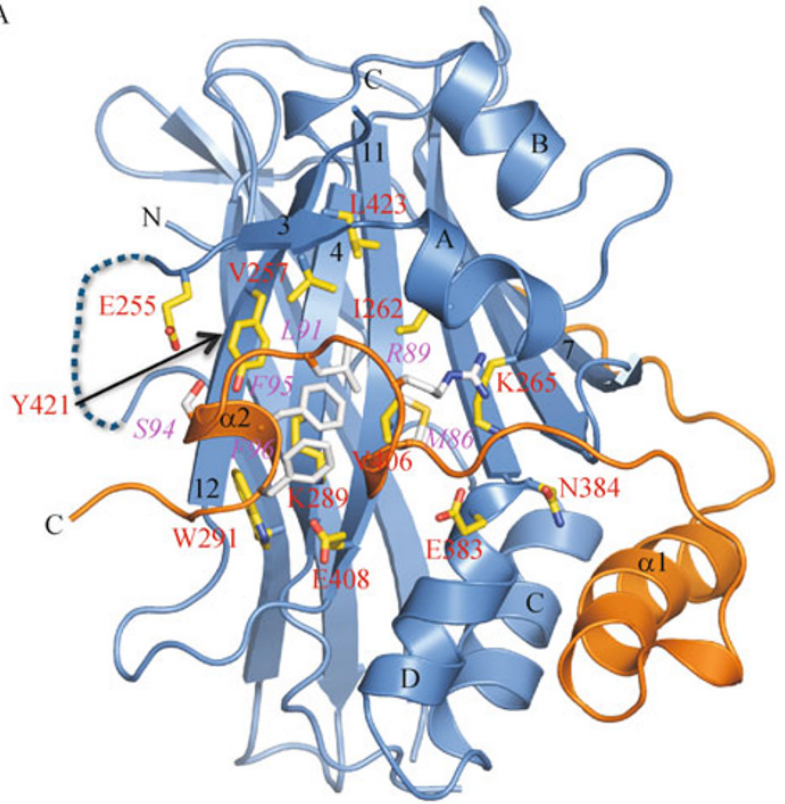

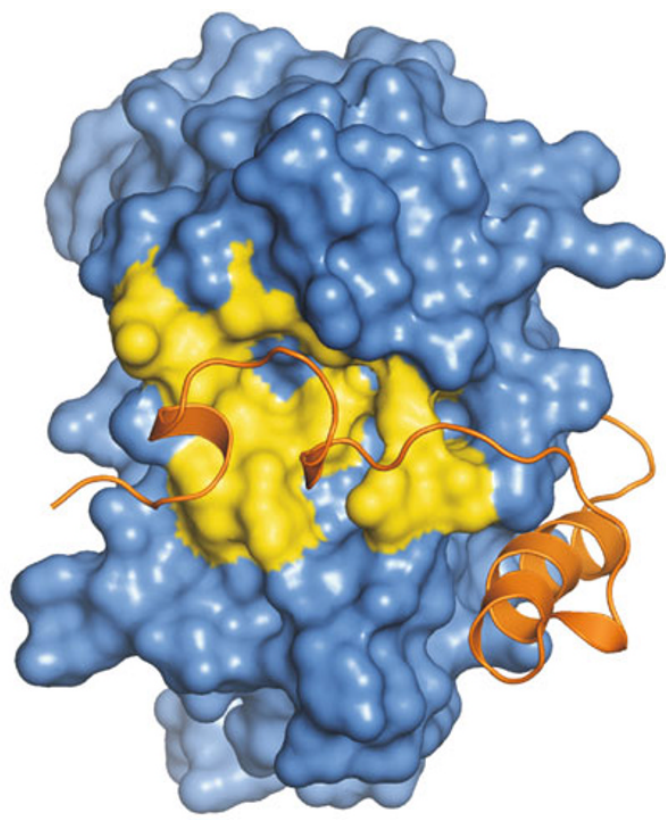

Figure 3. The YAP binding surface of TEAD. (A) Ribbon diagram of hTEAD1-YAP. The residues in TEAD that are critical for YAP binding are shown as yellow sticks and labeled in red, including E255, V257, I262, K265, K289, W291, E383, N384, V406, E408, Y421 and L423. The residues in YAP that are important for TEAD binding are shown as gray sticks and labeled in italic magenta, including M86, R89, L91, S94, F95 and F96. An arrow marks Y421 in TEAD1 mutated in Sveinsson's choriorentinal atrophy. (B) Molecular surface of TEAD1 YBD in the same orientation as in (A). Same residues in (A) that are deficient in YAP binding when mutated are colored in yellow. YAP TBD is shown in ribbons.

cells through blocking the YAP-dependent pro-proliferative and pro-survival gene expression program.

\section{REGULATION OF THE YAP-TEAD INTERACTION BY PHOSPHORYLATION}

LATS directly phosphorylates YAP at five HXRXXS consensus sites both in vitro and in vivo, including S61, S109, S127, S164 and S397 (Oh and Irvine, 2009; Zhao et al., 2009, 2010b). Phosphorylation at S127 creates a 14-3-3 binding site with the consensus of RXXpSXP. Binding of 14-3-3 to this site leads to cytoplasmic retention of YAP and its spatial separation from TEAD (Zhao et al., 2007; Oh and Irvine, 2008). Phosphorylation at S397 of YAP promotes the subsequent phosphorylation at $\mathrm{S} 400$ and possibly $\mathrm{S} 403$ by $\mathrm{CK} 1$, thus creating a phosphodegron with the consensus of DpSGXpS (Zhao et al., 2010b). This phosphodegron is recognized by $\beta T R C P$, leading to the polyubiquitination of YAP by SCF ${ }^{\beta T R C P}$ and its eventually degradation by the proteosome. The Hippo pathway thus regulates YAP through two mechanisms, cytoplasmic retention and ubiquitin-dependent proteolysis, both of which require phosphorylation of YAP at specific sites.

The mechanism by which 14-3-3 retains phospho-YAP in the cytoplasm is unclear at present. Again, several possible models exist. In the first model, 14-3-3 binding to YAP prevents TEAD from binding YAP by shielding the TEAD binding domain or creating steric hindrance or both. Along this vein, the 14-3-3 binding site S127 is in close proximity to YAP TBD (Fig. 2A). Furthermore, 14-3-3 exists as dimers and each 14-3-3 dimer has two binding sites for phospho-serinecontaining peptides. It has been proposed that one 14-3-3 monomer binds to an optimal phospho-serine site as a gatekeeper and the other monomer binds to an adjacent, suboptimal phospho-serine site (Yaffe, 2002). This bivalent binding mode between 14-3-3 and phospho-serine containing proteins greatly enhances their binding affinities and is likely to induce conformational changes of the proteins to mediate their biological function. Because YAP is phosphorylated at multiple sites, simultaneous engagement of two phosphoserine residues by a 14-3-3 dimer might physically shield YAP TBD away from TEAD or stabilize a conformation of YAP TBD (which is natively unfolded based on NMR data) that is incompatible for TEAD binding.

In the second model, 14-3-3 does not prevent TEAD from binding to phosphorylated YAP. The cytoplasmic retention of phospho-YAP is the result of a delicate balance between dynamic nuclear import and export. YAP does not have a nuclear localization signal (NLS) and needs to bind TEAD (which has an NLS) for nuclear import. 14-3-3 binding to phospho-YAP may shield the NLS of TEAD. Moreover, YAP has several putative nuclear export signals (NES). Phosphorylation of YAP may promote its nuclear export by Crm1 (Ren 
et al., 2010). 14-3-3 also contains an NES and may further enhance the nuclear export of phospho-YAP. The diminished nuclear import or enhanced nuclear export or both can explain the cytoplasmic retention of phospho-YAP by 14-3-3.

\section{CONCLUSIONS}

The evolutionarily conserved Hippo pathway controls tissue homeostasis in multicellular organisms by restricting cell proliferation and promoting cell death. Malfunction of the Hippo pathway results in hyperplasia and cancer. The YAPTEAD hybrid transcription factors are key downstream effectors of the Hippo pathway. In particular, the YAP-TEAD interaction is tightly regulated through YAP phosphorylation that is mediated by a kinase cascade in this pathway. Structural studies of the YAP-TEAD interaction have revealed the atomic details of this interaction and suggested a strategy for targeting this interaction with small molecules. How the YAP-TEAD interaction is regulated by phosphorylation and how 14-3-3 retains phospho-YAP in the cytoplasm remain significant, open questions in this field, however. Future structural and biochemical studies aimed at addressing these questions will undoubtedly advance our understanding of the molecular inner workings of this important tumor suppressor pathway and reveal nodes in this pathway that are potential points of pharmaceutical intervention.

\section{ACKNOWLEDGEMENTS}

The author thanks Hongtao Yu for critical reading of the manuscript. This work was supported by grant from the National Institutes of Health (to X.L. \#GM085004).

\section{ABBREVIATIONS}

CTGF, connective tissue growth factor; HCC, hepatocellular carcinomas; NES, nuclear export signal; NLS, nuclear localization signal; TBD, TEAD binding domain; YBD, YAP binding domain

\section{REFERENCES}

Alarcón, C., Zaromytidou, A.I., Xi, Q., Gao, S., Yu, J., Fujisawa, S., Barlas, A., Miller, A.N., Manova-Todorova, K., Macias, M.J., et al. (2009). Nuclear CDKs drive Smad transcriptional activation and turnover in BMP and TGF-beta pathways. Cell 139, 757-769.

Anbanandam, A., Albarado, D.C., Nguyen, C.T., Halder, G., Gao, X., and Veeraraghavan, S. (2006). Insights into transcription enhancer factor 1 (TEF-1) activity from the solution structure of the TEA domain. Proc Natl Acad Sci U S A 103, 17225-17230.

Asthagiri, A.R., Parry, D.M., Butman, J.A., Kim, H.J., Tsilou, E.T., Zhuang, Z., and Lonser, R.R. (2009). Neurofibromatosis type 2. Lancet 373, 1974-1986.

Badouel, C., Garg, A., and McNeill, H. (2009). Herding Hippos: regulating growth in flies and man. Curr Opin Cell Biol 21, 837-843.

Baumgartner, R., Poernbacher, I., Buser, N., Hafen, E., and Stocker, H. (2010). The WW domain protein Kibra acts upstream of Hippo in
Drosophila. Dev Cell 18, 309-316.

Cao, X., Pfaff, S.L., and Gage, F.H. (2008). YAP regulates neural progenitor cell number via the TEA domain transcription factor. Genes Dev 22, 3320-3334.

Chen, L., Chan, S.W., Zhang, X., Walsh, M., Lim, C.J., Hong, W., and Song, H. (2010). Structural basis of YAP recognition by TEAD4 in the hippo pathway. Genes Dev 24, 290-300.

Couture, J.F., Collazo, E., Ortiz-Tello, P.A., Brunzelle, J.S., and Trievel, R.C. (2007). Specificity and mechanism of JMJD2A, a trimethyllysine-specific histone demethylase. Nat Struct Mol Biol 14, 689-695.

Dong, J., Feldmann, G., Huang, J., Wu, S., Zhang, N., Comerford, S. A., Gayyed, M.F., Anders, R.A., Maitra, A., and Pan, D. (2007). Elucidation of a universal size-control mechanism in Drosophila and mammals. Cell 130, 1120-1133.

Edgar, B.A. (2006). From cell structure to transcription: Hippo forges a new path. Cell 124, 267-273.

Genevet, A., Wehr, M.C., Brain, R., Thompson, B.J., and Tapon, N. (2010). Kibra is a regulator of the Salvador/Warts/Hippo signaling network. Dev Cell 18, 300-308.

Grusche, F.A., Richardson, H.E., and Harvey, K.F. (2010). Upstream regulation of the hippo size control pathway. Curr Biol 20, R574-R582.

Grzeschik, N.A., Parsons, L.M., Allott, M.L., Harvey, K.F., and Richardson, H.E. (2010). Lgl, aPKC, and Crumbs regulate the Salvador/Warts/Hippo pathway through two distinct mechanisms. Curr Biol 20, 573-581.

Hao, Y., Chun, A., Cheung, K., Rashidi, B., and Yang, X. (2008). Tumor suppressor LATS1 is a negative regulator of oncogene YAP. J Biol Chem 283, 5496-5509.

Harvey, K., and Tapon, N. (2007). The Salvador-Warts-Hippo pathway- an emerging tumour-suppressor network. Nat Rev Cancer 7, 182-191.

Harvey, K.F., Pfleger, C.M., and Hariharan, I.K. (2003). The Drosophila Mst ortholog, hippo, restricts growth and cell proliferation and promotes apoptosis. Cell 114, 457-467.

Hisaoka, M., Tanaka, A., and Hashimoto, H. (2002). Molecular alterations of h-warts/LATS1 tumor suppressor in human soft tissue sarcoma. Lab Invest 82, 1427-1435.

Huang, J., Wu, S., Barrera, J., Matthews, K., and Pan, D. (2005). The Hippo signaling pathway coordinately regulates cell proliferation and apoptosis by inactivating Yorkie, the Drosophila Homolog of YAP. Cell 122, 421-434.

Jiménez-Velasco, A., Román-Gómez, J., Agirre, X., Barrios, M., Navarro, G., Vázquez, I., Prósper, F., Torres, A., and Heiniger, A. (2005). Downregulation of the large tumor suppressor 2 (LATS2/ KPM) gene is associated with poor prognosis in acute lymphoblastic leukemia. Leukemia 19, 2347-2350.

Justice, R.W., Zilian, O., Woods, D.F., Noll, M., and Bryant, P.J. (1995). The Drosophila tumor suppressor gene warts encodes a homolog of human myotonic dystrophy kinase and is required for the control of cell shape and proliferation. Genes Dev 9, 534-546.

Kango-Singh, M., Nolo, R., Tao, C., Verstreken, P., Hiesinger, P.R., Bellen, H.J., and Halder, G. (2002). Shar-pei mediates cell proliferation arrest during imaginal disc growth in Drosophila. Development 129, 5719-5730.

Kitagawa, M. (2007). A Sveinsson's chorioretinal atrophy-associated missense mutation in mouse Tead1 affects its interaction with the 
co-factors YAP and TAZ. Biochem Biophys Res Commun 361, 1022-1026.

Kosaka, Y., Mimori, K., Tanaka, F., Inoue, H., Watanabe, M., and Mori, M. (2007). Clinical significance of the loss of MATS1 mRNA expression in colorectal cancer. Int J Oncol 31, 333-338.

Lai, Z.C., Wei, X., Shimizu, T., Ramos, E., Rohrbaugh, M., Nikolaidis, N., Ho, L.L., and Li, Y. (2005). Control of cell proliferation and apoptosis by mob as tumor suppressor, mats. Cell 120, 675-685.

Li, Z., Zhao, B., Wang, P., Chen, F., Dong, Z., Yang, H., Guan, K.L., and $\mathrm{Xu}, \mathrm{Y}$. (2010). Structural insights into the YAP and TEAD complex. Genes Dev 24, 235-240.

Ling, C., Zheng, Y., Yin, F., Yu, J., Huang, J., Hong, Y., Wu, S., and Pan, D. (2010). The apical transmembrane protein Crumbs functions as a tumor suppressor that regulates Hippo signaling by binding to Expanded. Proc Natl Acad Sci U S A 107, 10532-10537.

Liu, A.M., Xu, M.Z., Chen, J., Poon, R.T., and Luk, J.M. (2010). Targeting YAP and Hippo signaling pathway in liver cancer. Expert Opin Ther Targets 14, 855-868.

Macias, M.J., Hyvönen, M., Baraldi, E., Schultz, J., Sudol, M., Saraste, M., and Oschkinat, H. (1996). Structure of the WW domain of a kinase-associated protein complexed with a prolinerich peptide. Nature 382, 646-649.

Minoo, P., Zlobec, I., Baker, K., Tornillo, L., Terracciano, L., Jass, J.R., and Lugli, A. (2007). Prognostic significance of mammalian sterile20-like kinase 1 in colorectal cancer. Mod Pathol 20, 331-338.

Nolo, R., Morrison, C.M., Tao, C., Zhang, X., and Halder, G. (2006). The bantam microRNA is a target of the hippo tumor-suppressor pathway. Curr Biol 16, 1895-1904.

Oh, H., and Irvine, K.D. (2008). In vivo regulation of Yorkie phosphorylation and localization. Development 135, 1081-1088.

Oh, H., and Irvine, K.D. (2009). In vivo analysis of Yorkie phosphorylation sites. Oncogene 28, 1916-1927.

Oh, H., and Irvine, K.D. (2010). Yorkie: the final destination of Hippo signaling. Trends Cell Biol 20, 410-417.

Oka, T., Mazack, V., and Sudol, M. (2008). Mst2 and Lats kinases regulate apoptotic function of Yes kinase-associated protein (YAP). J Biol Chem 283, 27534-27546.

Ota, M., and Sasaki, H. (2008). Mammalian Tead proteins regulate cell proliferation and contact inhibition as transcriptional mediators of Hippo signaling. Development 135, 4059-4069.

Overholtzer, M., Zhang, J., Smolen, G.A., Muir, B., Li, W., Sgroi, D.C., Deng, C.X., Brugge, J.S., and Haber, D.A. (2006). Transforming properties of YAP, a candidate oncogene on the chromosome 11q22 amplicon. Proc Natl Acad Sci U S A 103, 12405-12410.

Pan, D. (2007). Hippo signaling in organ size control. Genes Dev 21, 886-897.

Reddy, B.V., and Irvine, K.D. (2008). The Fat and Warts signaling pathways: new insights into their regulation, mechanism and conservation. Development 135, 2827-2838.

Ren, F., Zhang, L., and Jiang, J. (2010). Hippo signaling regulates Yorkie nuclear localization and activity through 14-3-3 dependent and independent mechanisms. Dev Biol 337, 303-312.

Robinson, B.S., Huang, J., Hong, Y., and Moberg, K.H. (2010). Crumbs regulates Salvador/Warts/Hippo signaling in Drosophila via the FERM-domain protein Expanded. Curr Biol 20, 582-590.

Seidel, C., Schagdarsurengin, U., Blümke, K., Würl, P., Pfeifer, G.P.,
Hauptmann, S., Taubert, H., and Dammann, R. (2007). Frequent hypermethylation of MST1 and MST2 in soft tissue sarcoma. Mol Carcinog 46, 865-871.

Steinhardt, A.A., Gayyed, M.F., Klein, A.P., Dong, J., Maitra, A., Pan, D., Montgomery, E.A., and Anders, R.A. (2008). Expression of Yesassociated protein in common solid tumors. Hum Pathol 39, 1582-1589.

Takahashi, Y., Miyoshi, Y., Takahata, C., Irahara, N., Taguchi, T., Tamaki, Y., and Noguchi, S. (2005). Down-regulation of LATS1 and LATS2 mRNA expression by promoter hypermethylation and its association with biologically aggressive phenotype in human breast cancers. Clin Cancer Res 11, 1380-1385.

Tao, W., Zhang, S., Turenchalk, G.S., Stewart, R.A., St John, M.A., Chen, W., and Xu, T. (1999). Human homologue of the Drosophila melanogaster lats tumour suppressor modulates CDC2 activity. Nat Genet 21, 177-181.

Tapon, N., Harvey, K.F., Bell, D.W., Wahrer, D.C., Schiripo, T.A., Haber, D.A., and Hariharan, I.K. (2002). salvador Promotes both cell cycle exit and apoptosis in Drosophila and is mutated in human cancer cell lines. Cell 110, 467-478.

Thompson, B.J., and Cohen, S.M. (2006). The Hippo pathway regulates the bantam microRNA to control cell proliferation and apoptosis in Drosophila. Cell 126, 767-774.

Tian, W., Yu, J., Tomchick, D.R., Pan, D., and Luo, X. (2010). Structural and functional analysis of the YAP-binding domain of human TEAD2. Proc Natl Acad Sci U S A 107, 7293-7298.

Varelas, X., Sakuma, R., Samavarchi-Tehrani, P., Peerani, R., Rao, B. M., Dembowy, J., Yaffe, M.B., Zandstra, P.W., and Wrana, J.L. (2008). TAZ controls Smad nucleocytoplasmic shuttling and regulates human embryonic stem-cell self-renewal. Nat Cell Biol 10, 837-848.

Wu, S., Huang, J., Dong, J., and Pan, D. (2003). hippo encodes a Ste20 family protein kinase that restricts cell proliferation and promotes apoptosis in conjunction with salvador and warts. Cell 114, 445- 456.

Wu, S., Liu, Y., Zheng, Y., Dong, J., and Pan, D. (2008). The TEAD/ TEF family protein Scalloped mediates transcriptional output of the Hippo growth-regulatory pathway. Dev Cell 14, 388-398.

Xu, T., Wang, W., Zhang, S., Stewart, R.A., and Yu, W. (1995). Identifying tumor suppressors in genetic mosaics: the Drosophila lats gene encodes a putative protein kinase. Development 121, 1053-1063.

Yaffe, M.B. (2002). How do 14-3-3 proteins work?- Gatekeeper phosphorylation and the molecular anvil hypothesis. FEBS Lett 513, 53-57.

Yu, J., Zheng, Y., Dong, J., Klusza, S., Deng, W.M., and Pan, D. (2010). Kibra functions as a tumor suppressor protein that regulates Hippo signaling in conjunction with Merlin and Expanded. Dev Cell 18, 288-299.

Zender, L., Spector, M.S., Xue, W., Flemming, P., Cordon-Cardo, C., Silke, J., Fan, S.T., Luk, J.M., Wigler, M., Hannon, G.J., et al. (2006). Identification and validation of oncogenes in liver cancer using an integrative oncogenomic approach. Cell 125, 1253-1267.

Zeng, Q., and Hong, W. (2008). The emerging role of the hippo pathway in cell contact inhibition, organ size control, and cancer development in mammals. Cancer Cell 13, 188-192.

Zhang, J., Ji, J.Y., Yu, M., Overholtzer, M., Smolen, G.A., Wang, R., Brugge, J.S., Dyson, N.J., and Haber, D.A. (2009a). YAP-dependent 
induction of amphiregulin identifies a non-cell-autonomous component of the Hippo pathway. Nat Cell Biol 11, 1444-1450.

Zhang, L., Yue, T., and Jiang, J. (2009b). Hippo signaling pathway and organ size control. Fly (Austin) 3, 68-73.

Zhao, B., Wei, X., Li, W., Udan, R.S., Yang, Q., Kim, J., Xie, J., Ikenoue, T., Yu, J., Li, L., et al. (2007). Inactivation of YAP oncoprotein by the Hippo pathway is involved in cell contact inhibition and tissue growth control. Genes Dev 21, 2747-2761.

Zhao, B., Lei, Q.Y., and Guan, K.L. (2008a). The Hippo-YAP pathway: new connections between regulation of organ size and cancer. Curr Opin Cell Biol 20, 638-646.

Zhao, B., Ye, X., Yu, J., Li, L., Li, W., Li, S., Yu, J., Lin, J.D., Wang, C.Y.,
Chinnaiyan, A.M., et al. (2008b). TEAD mediates YAP-dependent gene induction and growth control. Genes Dev 22, 1962-1971.

Zhao, B., Kim, J., Ye, X., Lai, Z.C., and Guan, K.L. (2009). Both TEADbinding and $\mathrm{WW}$ domains are required for the growth stimulation and oncogenic transformation activity of yes-associated protein. Cancer Res 69, 1089-1098.

Zhao, B., Li, L., Lei, Q., and Guan, K.L. (2010a). The Hippo-YAP pathway in organ size control and tumorigenesis: an updated version. Genes Dev 24, 862-874.

Zhao, B., Li, L., Tumaneng, K., Wang, C.Y., and Guan, K.L. (2010b). A coordinated phosphorylation by Lats and CK1 regulates YAP stability through SCF(beta-TRCP).Genes Dev 24, 72-85. 